\title{
DE L'APPROCHE COMMUNICATIVE À L'APPROCHE ACTIONNELLE : LA COMPETENCE DE LECTURE À HAUTE VOIX À TRAVERS LES MANUELS
}

Dans le cadre du présent article, nous avons essayé de cerner une éventuelle évolution quant à la façon d'aborder l'activité de lecture à haute voix dans des manuels représentatifs de l'Approche Communicative et de l'Approche Actionnelle. Nous avons recherché si et comment la compétence de lecture est exploitée dans ces manuels, si l'activité de lecture à haute voix y figure, ainsi que la typologie des activités et les conseils méthodologiques proposés par les guides pédagogiques pour l'exploitation de la lecture à haute voix. Les résultats auxquels nous sommes parvenue démontrent que ce type de lecture est très rarement proposé de manière explicite par les manuels des approches mentionnées ci-dessus et accompagne seulement l'exploitation d'extraits de textes littéraires.

Mots-clés : lecture expressive, Approche Communicative, Approche Actionnelle, manuels, états des lieux, compétence phonologique, éléments suprasegmentaux

\section{Introduction}

Si nous avons entrepris cette enquête, c'est parce que la lecture à haute voix nous tient à cœur depuis le temps où notre professeur de FLE nous demandait de lire à chacun de nous, un petit paragraphe du texte qui servait de trame pour le développement des compétences qu'il s'était fixées comme objectif d'enseignement. Lorsqu'il nous lisait des extraits de textes, littéraires la plupart du temps, la mélodie de sa voix et la façon dont il accentuait les syllabes pour les mettre en relief réveillaient en nous des sentiments divers et variés, nous faisaient vivre le texte, nous introduisaient dans d'autres personnalités qui nous faisaient rêver.

Une autre raison pour laquelle nous avons entrepris cette enquête, c'est que nous nous sommes aperçue que nos étudiants et futurs enseignants avaient

\footnotetext{
${ }^{1}$ mapateli@frl.uoa.gr
} 
eux-mêmes des difficultés à lire, des difficultés d'ordre prosodique surtout, et qu'ils avaient besoin d'être formés à lire et à faire lire, soit par le biais d'ouvrages appropriés soit en suivant des journées de formation.

Quelques définitions de la lecture à haute voix et de la lecture expressive nous permettront d'expliquer leur importance dans l'enseignement du FLE et les raisons pour lesquelles nous avons centré notre recherche sur ce type de lecture.

La lecture à haute voix est un processus complexe et complet qui requiert une aptitude visuelle, orthographique, linguistique, sémantique et cognitive. Elle implique trois opérations : une lecture silencieuse visuelle qui permet la compréhension du document (je lis, je comprends), une oralisation du texte écrit (je dis ce que j'ai lu et compris) et une opération de rétroaction (l'effet produit par la diction du lecteur soit sur lui-même, soit sur son auditoire). La lecture orale est un processus durant lequel le lecteur voit des signes écrits, les transforme en sons et c'est à partir de ces sons qu'il s'écoute prononcer, il construit du sens : le lecteur se transforme en auditeur du message écrit. Dans le cas de la lecture orale, l'oralisation précède et permet la compréhension alors que dans le cas de la lecture à haute voix, la compréhension précède et contribue à la bonne qualité de l'oralisation (BEAUME 1987). Dans la lecture à haute voix, le lecteur visualise, comprend, interprète et prononce le message écrit. La lecture orale devient un moyen pour la compréhension, la lecture à haute voix, un moyen de communication partagée.

Par ailleurs, la lecture à haute voix est une forme dynamique de l'oral, une activité créative puisque le lecteur est appelé à transmettre du sens tel qu'il l'a interprété au fur et à mesure qu'il lit le texte, un sens habillé d'une musique qui dépend de cette interprétation, une interprétation qui « oriente et conditionne [...] la compréhension des auditeurs » puisque " lire à haute voix exige de vocaliser significativement chaque mot, phrase, paragraphe dans son contexte discursif » (REYZABAL 2015 : 2). Le lecteur qui lit à voix haute devient le médiateur des idées et des sentiments de l'auteur du texte tels qu'il les perçoit et les interprète. Dans ce sens, lire c'est oraliser « le langage utilisé par un autre » (REYZABAL 2015 : 2) et rendre compte de son style. On est loin de l'activité mécanique décriée par le CECRL « Les activités les plus mécaniques de conservation du sens (la répétition, la dictée, la lecture à haute voix, la transcription phonétique) sont actuellement décriées dans un enseignement orienté vers la communication parce qu'elles sont artificielles et ont des effets en retour considérés comme peu souhaitables ». (CECRL 2001 : 80). La lecture à haute voix n'est pas mécanique. Tout dépend de la façon et de la raison pour laquelle on l'utilise.

L'activité de la lecture à haute voix telle que nous l'avons décrite plus haut, orientée vers celui qui reçoit le message plus que vers le lecteur lui- 
même, est une lecture fonctionnelle. Selon (BEAUME 1987 : 3) «ce qui caractérise la situation de lecture et la rend fonctionnelle, c'est que l'auditoire attend l'information que le lecteur va donner, à condition, bien sûr, qu'il n'ait pas le texte sous les yeux ce qui aurait pour résultats de faire disparaître cette fonctionnalité ».

De plus, la musicalité de la voix porteuse de sens renvoie à la mélodie, au rythme, aux pauses, à l'accentuation, à tous ces éléments prosodiques qui doivent être appris et enseignés, qui diffèrent d'une langue à une autre, qui donnent à l'écriture plate du relief, du sentiment. La lecture à haute voix est le moyen idéal pour entraîner les élèves à perfectionner leur élocution, à produire une prononciation correcte à partir de la forme écrite des mots en d'autres termes, à jongler entre la compétence orthoépique et la compétence orthographique.

La compétence de lecture à haute voix dans sa dimension orthoépique et communicative est importante pour l'apprentissage du FLE dans la mesure où l'élève doit apprendre à décoder ce qu'il voit écrit selon les règles de la chaîne sonore, le comprendre, le ressentir et le communiquer en classe comme il l'aurait fait dans la vie quotidienne et en langue maternelle.

Lire à haute voix, c'est mettre au premier plan toutes ces qualités articulatoires et prosodiques qui transforment les lettres en sons, transmettent du sens à l'oral, améliorent la production orale de l'élève en lui permettant de communiquer une information et prendre plaisir à ça, capter/accrocher l'attention de son auditeur. Ce qui rejoint l'avis d'Estelle Riquois qui postule que « lorsque l'élève lit le texte à voix haute, ce qui peut aussi être nommé lecture active, il ne s'agit pas simplement d'oraliser le texte, de le déchiffrer à un niveau sonore qui permet à tous de l'entendre. Il s'agit plutôt de proposer une lecture qui associe la compréhension et l'interprétation. La lecture à voix haute pensée de manière active est une lecture qui nécessite l'adaptation de l'élève au texte qu'il lit, qui demande de la créativité et qui permet de partager sa lecture et le plaisir de lire » (RIQUOIS 2019 : 154).

L'enseignement d'une telle lecture est difficile sans support pédagogique adéquat et sans formation appropriée.

C'est cette lecture active que nous avons voulu voir si elle existait dans ces deux catégories de manuels, un type de lecture qui se rapproche de la lecture expressive. Notre recherche nous a permis de faire l'état des lieux de la façon dont l'activité de lecture à haute voix est traitée dans des manuels appartenant à deux approches didactiques qui sans être très éloignées dans le temps sont assez différentes dans leur façon de l'aborder.

Dans le processus d'enseignement du FLE selon les principes de l'Approche Communicative (désormais AC) et surtout jusque dans les années 1980, la lecture à haute voix était pratiquée assez couramment dans les 
classes, ensuite, cette pratique disparaît parce que la qualité de la production orale du message et de ses aspects phonologiques, présente dans les objectifs d'enseignement des méthodologies précédentes, est sacrifiée au profit du sens transmis par le message et, de plus, parce qu'une telle compétence nécessitait et nécessite d'ailleurs un enseignement de tous les aspects phonologiques qui la sous-tendent ce qui exigeait un effort considérable de la part des élèves mais aussi un savoir de la part des enseignants, un savoir qui tombait petit à petit en désuétude.

Après les années 1990, toujours en pleine période d'Approche Communicative, pendant laquelle la première série des manuels que nous étudions a été conçue et éditée, la pratique de la lecture en classe de langue signifie apprendre à construire du sens tout en développant des compétences grammaticales, syntaxiques et interculturelles. Cette nouvelle vision de la lecture se traduit par une nouvelle manière d'aborder l'activité de lecture en classe de langue et par une implication active et consciente de l'élève/ lecteur dans l'acte de lire. Toutefois, comme nous le verrons par l'étude des manuels mentionnés ci-dessous, cette lecture est de type indéterminé, la consigne " lisez » apparaît rarement dans le livre de l'élève, ou n'apparaît pas du tout et sa fonction est la compréhension du texte par l'élève/lecteur et le développement de diverses compétences telles la compréhension des écrits et la production écrite et/ou orale. Même si, comme le disent les spécialistes, ce qui est également confirmé par notre expérience d'enseignante de FLE dans ces temps là, la lecture à haute voix « redevient une activité récurrente souvent ritualisée qui appartient à la pratique quotidienne des enseignants qui suivent l'approche communicative » (RIQUOIS 2019 : 154), la consigne « lisez à haute voix » est absente des manuels représentatifs de cette époque, du moins de ceux qui ont servi de base à notre étude, et la lecture à haute voix devient entre les mains de l'enseignant un moyen pour faire développer différentes stratégies de lecture qui correspondent aux différents types de compréhension. La lecture à haute voix que nous faisions faire à nos élèves était une lecture « linéaire », deuxième étape après une première lecture silencieuse (collective ou individuelle, d'après les guides pédagogiques) qui contribuait à la vérification des hypothèses de compréhension, à l'interprétation du contenu du texte, à la réalisation des activités l'accompagnant. La lecture expressive n'était pas proposée puisqu' elle se fonde sur la compétence phonologique qui était délaissée des objectifs d'enseignement de cette période-là.

Cet acte de lire se transforme en action de lire dans l'Approche Actionnelle (désormais AA) où le lecteur agit/lit dans le but de traduire une tâche quotidienne et de communiquer le sens que lui-même a perçu et compris au moyen du texte lu. La lecture-compréhension évolue en lecturecommunication, la lecture silencieuse est complétée par une lecture à haute 
voix et le texte, vivant et non découpé de son contexte réel, est lu dans un objectif communicatif précis.

Nos hypothèses de recherche que nous vérifierons au moyen de notre enquête s'articulent autour des points suivants :

a) il y aurait une évolution quant à la façon d'aborder l'activité de lecture à haute voix entre ces deux méthodologies ;

b) l'activité de lecture à haute voix dans sa dimension expressive rejoindrait les principes de l'Approche Actionnelle plus que ceux de l'Approche Communicative dont elle est la suite, (l'acte de lire se transforme en action de lire) et de ce fait elle devrait être présente dans les manuels utilisés pour l'enseignement du FLE selon l'Approche Actionnelle (consigne+contenu, en d'autres termes, que l'on trouverait des consignes invitant les élèves à lire à haute voix et que le contenu des activités ferait développer l'expressivité de la production orale des élèves) ;

c) les textes littéraires seraient accompagnés d'activités de lecture expressive ;

d) les activités de lecture à haute voix inviteraient les élèves à développer et à se servir de la compétence phonologique puisque dans sa dimension communicative, la lecture à haute voix présuppose la bonne qualité sonore du message communiqué.

\section{Outil de recherche}

Si nous avons choisi de faire l'état des lieux sur la compétence de lecture à haute voix à travers les manuels, c'est parce que le manuel est un « outil privilégié de la formation des enseignants » (RIQUOIS 2010 : 248), un support de cours pour les apprenants, une aide à sa préparation pour les enseignants, avec des objectifs et des activités préparées et conçues par des didacticiens instruits selon les principes méthodologiques dont se réclament les manuels.

De son côté, le guide pédagogique qui complète les manuels offre des propositions didactiques récentes et constitue un outil d'autoformation surtout pour ceux qui n'ont ni le temps, ni la possibilité de suivre des séminaires de formation. Dans ce sens, le choix du manuel et de son guide pédagogique informe sur les pratiques des enseignants qui les ont choisis et s'en servent. L'absence ou la présence de l'activité de lecture à haute voix dans les manuels est la preuve d'une ligne directrice pour l'enseignement du FLE et oriente les objectifs d'enseignement des enseignants. 


\section{Corpus/public/niveau}

Pour que notre corpus de recherche soit le plus représentatif possible même pour ce début d'enquête, nous avons choisi trois manuels utilisés pour l'enseignement du FLE selon les principes de l'Approche Communicative rédigés entre 1988 et 1996 et trois manuels utilisés pour l'enseignement du FLE selon les principes de l'Approche Actionnelle, rédigés entre 2013 et 2017.

Le public choisi : grands adolescents et adultes.

Le niveau visé par les manuels enseignant le FLE selon l'Approche Actionnelle est le $\mathrm{B} 1$ ce qui correspondrait à un niveau de préparation au DELF $1^{\text {er }}$ degré pour les manuels de l'Approche Communicative, selon le site de l'IFA. ${ }^{2}$

Étant donné que notre enquête porte sur la lecture à haute voix dans sa dimension expressive, nous avons pensé que le public mentionné ci-dessus aurait un savoir socioculturel, un niveau de compétences communicatives et langagières et une maturité qui lui permettraient d'affronter la difficulté linguistique (tant sur le plan de la morphosyntaxe que sur celui de la prononciation) et la thématique socioculturelle des textes proposés dans les manuels étudiés. Nous avons également pensé que, vu l'âge et le niveau, et le fait que ces textes à partir de ce niveau se prêtaient à l'apprentissage de la langue et de sa culture, les enseignants feraient de la lecture un objectif d'enseignement dans leurs cours. Pourtant, selon une enquête que nous avons effectuée et dont les résultats ont été communiqués lors du $3^{\mathrm{e}}$ Congrès européen des professeurs de français ${ }^{3}$, plus on montait en niveau selon les prescripteurs du CECRL, plus le temps consacré par les enseignants à la lecture à haute voix baissait.

Consacrez-vous du temps à l'enseignement de la lecture à haute voix au niveau :

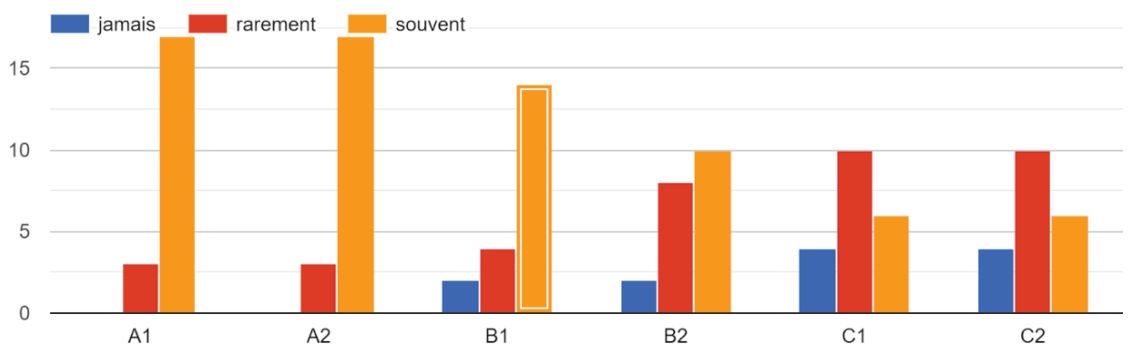

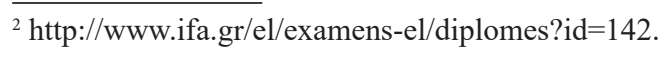

${ }^{3}$ Dont le graphique ci-dessous. 
Les manuels choisis pour la réalisation de notre enquête sont les suivants : Le NOUVEL ESPACES 2 (éditions Hachette), LE NOUVEAU SANS FRONTIERES 2 (éditions CLE International), Panorama 2 (éditions CLE International), conçus selon les principes de l'Approche Communicative, et Tendances B1 (éditions CLE International), Écho B1.1 et Écho B1.2 (éditions CLE International), LE NOUVEL édito Niveau B1 (éditions Didier), conçus selon les principes de l'Approche Actionnelle.

\section{La place de la lecture à haute voix} dans les manuels appartenant à l'Approche Communicative

Aucun des trois manuels que nous avons étudiés appartenant à l'Approche Communicative ne contient dans le livre de l'élève de consigne appelant les élèves à une lecture à haute voix. La consigne courante dans les livres de l'élève des trois manuels, lorsque celle-ci y figure, est « lisez », le type de lecture n'est pas mentionné et renvoie à une lecture individuelle, collective, silencieuse implicitement proposée dans LE NOUVEAU SANS FRONTIERES 2 et dans PANORAMA 2 (on ne trouve pas la qualification « silencieuse ») alors que dans LE NOUVEL ESPACES 2 le type de lecture silencieuse est explicitement proposé dans le guide pédagogique. Ce type de lecture renvoie à des activités d'interprétation/compréhension et d'analyse du contenu du texte, individuelles ou collectives. La lecture silencieuse devient le moyen pour le développement des différentes stratégies de lecture afin de parvenir aux différents types de compréhension des écrits et de développer des compétences communicatives et langagières tant à l'oral qu'à l'écrit.

On s'attendrait à ce que l'exploitation des textes littéraires, qui figurent dans ces manuels, inclue la lecture à haute voix, pourtant là encore le type de lecture associé est la lecture silencieuse, individuelle, proposée explicitement (LE NOUVEL ESPACES 2) ou implicitement (LE NOUVEAU SANS FRONTIERES 2 et PANORAMA 2). À trois exceptions près, deux poèmes et un extrait d'une pièce de théâtre qui invitent (une fois explicitement et deux fois implicitement) à une lecture à haute voix. Il s'agit d'un texte par manuel.

a) Un poème de Raymond Queneau, « L'espèce humaine », pour lequel les auteurs proposent aux élèves une lecture rapide et individuelle pour sa compréhension et une lecture à haute voix une fois qu'ils ont compris le sens du poème : " Ne lire ou ne faire lire à haute voix que lorsque les étudiants en ont élucidé le sens général » (LE NOUVEL ESPACES 2, guide : 45). C'est la seule fois où le mot lecture à haute voix apparaît. Les autres poèmes sont lus de manière rapide, silencieuse ou sans précision particulière sur le type de lecture et écoutés «Faire lire le poème silencieusement, puis faire écouter 
l'enregistrement. Distribuer les questions à des groupes différents. Correction orale collective » (LE NOUVEL ESPACES 2, guide : 87).

b) Un poème de Jacques Prévert, « Le message », dont la consigne est assez ambigüe, laisse des interprétations diverses et ambivalentes et inviterait implicitement à une lecture à haute voix. "Mimez chaque vers de ce poème " (LE NOUVEAU SANS FRONTIERES 2, livre de l'élève : 63), qui renverrait à une récitation, présuppose une lecture expressive donc à haute voix, fondée sur une bonne articulation et une accentuation expressive qui traduit le genre du message et les sentiments de son auteur. Par ailleurs, « mimez» supposerait que l'élève mime la lecture de l'enseignant qui lui sert de modèle phonologique. Dans ce sens, l'apprenant lit, voire répète, voire « mime » le sentiment tel qu'il est interprété et proposé par l'enseignant, avec son débit à lui, son intonation à lui, la mélodie que lui donne au vers. Ce que la consigne ne nous permet pas de comprendre, c'est si l'élève répète en lisant ou s'il répète en produisant sans support écrit. Dans la mesure où il lit en répétant à partir du modèle de son enseignant, on parle de lecture/répétition et l'apprenant est en position sécurisante : sa production n'est pas mal jugée par ses camarades étant donné que la lecture n'est pas le fruit de sa propre interprétation qui risque de ne pas être la bonne. Dans ce sens, il n'y a pas d'interaction entre le texte et l'élève/lecteur. En d'autres termes, la lecture du poème n'est pas active. Nous pouvons parler d'un acte de lire. Une autre interprétation du verbe « mimer» a affaire avec la gestuelle et dans ce sens, l'activité inviterait l'élève à faire les gestes qui correspondent au sens du poème. Aucun conseil relatif à la lecture à haute voix n'est présent dans le guide pédagogique.

c) Un extrait d'une pièce de théâtre de Courteline, « Le commissaire est bon enfant » (Panorama 2 : 28-29) dont l'étude des traits de caractère des personnages, l'intonation caractérisant leurs propos, les gestes et les déplacements correspondants constituent un travail préparatif à une lecture expressive/interprétation théâtrale qui peut être mise en scène et conduire à un jeu de rôles qui n'est pas loin d'une interprétation théâtrale. Le geste et le mouvement renvoient au rythme, le rythme à la prosodie et la prosodie à la lecture expressive, au jeu de rôles.

Pour l'Approche Communicative, les activités communicatives les plus authentiques sont « les jeux de rôle, les simulations, les résolutions de problèmes etc. La lecture à haute voix rentre dans la catégorie des activités non communicatives comme les exercices structuraux ou les exercices de simple répétition » (GERMAIN $2001: 211$ cité par LAH 2013 : 203), et sert plutôt de moyen pour le développement des compétences linguistiques. 


\section{La place de la lecture à haute voix dans les manuels appartenant à l'Approche Actionnelle}

Dans les trois manuels relevant de l'Approche Actionnelle, aucune consigne n'appelle les élèves à lire à haute voix, du moins explicitement. Les consignes que l'on y trouve, "Lisez », " Lecture du document, du tract de l'article », etc., accompagnent l'exploitation du texte proposé et renvoient à une lecture silencieuse qui n'est pas mentionnée explicitement non plus.

Cette lecture silencieuse aboutit à la réalisation d'activités associées aux textes lus et vise à la compréhension des écrits (les différents types de compréhension/globale, détaillée, sélective, fine renvoyant aux différentes stratégies de lecture), au développement de la compétence linguistique (lexicale, grammaticale, prosodique), à la production orale et/ou écrite, au développement de l'esprit de synthèse et du résumé, à la capacité d'ordonner les idées.

La typologie des activités accompagnant les lectures consiste en des questions à réponses ouvertes libres ou guidées par le contenu du texte donc en des activités de production orale, des activités à trous, de classement, d'association, mixtes (compréhension+production) et conçues assez souvent selon les exigences des examens du DELF.

À la différence des activités relevant de l'Approche Communicative, au moyen des activités relevant de l'Approche Actionnelle, les élèves apprennent à utiliser ce qu'ils ont appris, dans le cadre d'une tâche de vie quotidienne.

Bien que la lecture à haute voix soit absente des consignes et des objectifs des manuels, implicitement elle est présente et dépend de l'utilisation qu'en fera l'enseignant pour atteindre les objectifs visés par certaines activités. La lecture à haute voix implicite est associée aux textes littéraires que l'on trouve dans les manuels.

Dans Écho B1.1, il n'y a pas de consigne incitant à une lecture à haute voix, ce qui est le cas dans Écho B1.2, où à deux endroits (page 65 : extrait d'un film ; page 128 : extrait d'une pièce de théâtre) les activités invitent à une préparation implicite à la lecture expressive. Pour ce qui est de l'extrait du film, les élèves lisent chaque phrase, et sont appelés à comprendre et à caractériser le sentiment qu'elles expriment et le ton sur lequel elles auraient pu être dites. Cette étape de compréhension sélective pourrait être complétée par une activité de lecture expressive qui n'a pourtant pas été proposée.

Il en est de même pour les deux extraits de pièces de théâtre que l'on trouve dans Écho B1.2 et dans LE NOUVEL édito 1. Dans l'extrait d'une pièce de théâtre écrite par Rolland Dubillard «Les Diablogues et autres inventions à deux voix » (Écho B1.2 : 128), l'activité invite les élèves à imaginer une mise en scène pour le dialogue, à se transformer en metteurs en scène et à 
mettre en scène les deux acteurs participant à cet extrait, en focalisant sur l'intonation des phrases, les gestes et les déplacements des personnages (entre parenthèses dans la consigne). La consigne et l'explication donnée entre parenthèses invitent les élèves à travailler la compétence phonologique et plus précisément la composante prosodique (intonation, accentuation, rythme) et à mettre en relation la mélodie de la voix et le rythme de la phrase lue avec le geste et le mouvement du corps. Voilà une activité actionnelle qui s'oppose au caractère statique et à l'immobilisme d'une lecture mécanique. Les élèves lisent le dialogue en bougeant dans la classe, en gesticulant, en rythmant leur phrase, en prenant des risques d'interprétation, en mettant en valeur leurs sentiments par le biais de l'accentuation expressive avant de le jouer. Comme l'a dit Riquois « La structure de la classe est bouleversée. La communication ne passe plus uniquement par la langue mais fait intervenir le corps en libérant la parole des apprenants » (RIQUOIS 2010 : 250). Il en est de même dans le manuel Tendances qui foisonne en textes littéraires et en activités de lecture. Aucune lecture à haute voix n'est pourtant proposée. Il y a tout de même quatre endroits dans le manuel, deux extraits de pièces de théâtre et deux extraits de films, pour lesquels les auteurs auraient pu proposer une lecture à haute voix et même une lecture expressive. Les activités qui encadrent les textes incitent les apprenants à un travail de compréhension et de préparation au niveau de la kinésique, de la prosodie, et des expressions $\mathrm{du}$ visage traduisant les sentiments des protagonistes (groupes rythmiques, accentuation, intonation) qui pourrait se terminer par une lecture expressive, dernière étape avant l'activité d'interprétation théâtrale (Tendances : " Découper la chaîne sonore », "S'aider de l'intonation », " Distinguer les sons proches » (p. 16) ; "Lisez le sketch. Caractérisez chaque personnage avec les mots ci contre ». "Par deux jouez le sketch. Trouvez les gestes, les intonations et les expressions du visage ». " Jeu de rôles. Par deux jouez une des scènes suivantes » (p. 52) ; "Lisez la scène ». " Voici les moments d'une dispute. Remettez les dans l'ordre » (p. 67) ; «Lisez la scène du film... Répartissez-vous les deux rôles ». «Avec votre partenaire, imaginez la fin de la scène. Jouez-la devant la classe ». (p. 95)).

Même si la lecture théâtrale rapporte une situation éloignée des interactions que l'on est susceptible de rencontrer dans la vie quotidienne, même si la forme d'expression ne contient pas les spécificités de l'oral, même si la diction paraît peu naturelle (MORAZ, PRIKHODKINE 2011 : 108), elle est active, elle implique les apprenants dans une interaction où le corps et le langage s'allient pour transformer un texte écrit en document sonore authentique. Donc, le projet de mise en scène prépare les élèves à une performance orale qui tient compte d'une lecture à haute voix fondée sur l'intonation expressive. 
La lecture à haute voix est également absente dans LE NOUVEL édito même quand il s'agit de textes littéraires ou de poèmes à une exception près : l'extrait de la pièce de théâtre de Jean-Michel Ribes, Musée haut, musée bas, Actes Sud, 2004 (LE NOUVEL édito 1 : 147) où les auteurs demandent explicitement aux élèves de faire une lecture expressive. Les auteurs proposent la compréhension des écrits au moyen d'une lecture silencieuse qui conduit à une production orale. La production orale consiste en une lecture expressive, donc à haute voix, de l'extrait de la pièce de théâtre qui prépare les élèves à l'acquisition de la compétence de lecture. Les auteurs les invitent à effectuer par deux une lecture expressive de la pièce. "Par deux, faites une lecture expressive de ce dialogue ». En d'autres termes, les élèves sont appelés à lire par deux le dialogue de la pièce donc à interpréter des situations et des sentiments sans qu'un travail de préparation à cette performance ne soit proposé (des activités ciblées sur l'accentuation expressive, le rythme, l'intonation adéquate au contenu) puisque les activités de compréhension qui précèdent ne préparent en aucun cas cette lecture expressive. Les propositions du guide pédagogique insistent toutefois sur les points qui déterminent la bonne qualité de la lecture sans expliquer pourtant comment et par quelles activités y parvenir : " [en binômes, puis s'exprimer devant la classe] Afin de faire une lecture expressive de cet extrait littéraire, les apprenants ne doivent plus avoir de problème de déchiffrage. Le texte doit avoir été bien préparé et son sens doit être clair. Bien veiller à l'articulation, la ponctuation, l'intonation, au rythme et aux liaisons. Possibilité de faire jouer cette courte scène en classe par plusieurs groupes. Puis, échanger sur les différentes interprétations de chacun » (LE NOUVEL édito 1, guide pédagogique : 156).

Pour les autres textes littéraires que l'on trouve dans le même manuel, après la lecture qui permet la compréhension du document, la production orale consiste en des réponses libres à des questions relatives au sujet. Aucun travail sur l'accentuation, le rythme et l'intonation qui sous-tendent une lecture expressive et une récitation du poème de Musset absentes des productions orales proposées dans le manuel (livre de l'élève : 80). Il en est de même pour l'exploitation du texte de Jules Verne (livre de l'élève : 95), et pour celle du texte de Perec (livre de l'élève : 111) ou celle du texte de Rufin (livre de l'élève : 167).

\section{Conclusion}

Aussi bien dans les manuels relevant de l'Approche Communicative que dans ceux de l'Approche Actionnelle on trouve seulement deux appels explicites à une lecture à haute voix. L'un concerne un poème et on le trouve 
dans les manuels relevant de l'Approche Communicative et l'autre, un extrait de pièce de théâtre et on le trouve dans les manuels relevant de l'Approche Actionnelle. Dans le cadre de cette dernière, l'exploitation prosodique, assez superficielle mais moins que dans l'AC, des extraits de pièces de théâtre et de films conduit implicitement à une lecture expressive nécessaire à la représentation théâtrale proposée par les activités du manuel. En effet, les activités qui encadrent ces textes portent sur l'articulation correcte des sons et sur les éléments suprasegmentaux qui sous-tendent une lecture à haute voix expressive. Nous pourrions donc parler d'une évolution quant à la façon d'aborder l'activité de lecture puisqu'il y a un effort de la part des concepteurs des manuels selon l'AA de travailler sur la compétence phonologique et de la considérer comme support fondamental pour une lecture à haute voix réussie.

Pour ce qui est du nombre des activités interpelant à une lecture à haute voix, celui-ci est restreint aussi bien dans les manuels relevant de l'AC que dans ceux de l'AA.

Le type de textes auquel sont reliés des appels implicites ou explicites à une lecture à haute voix sont des textes littéraires (pièce de théâtre et poèmes) et des extraits de films et on les trouve aussi bien dans les manuels relevant de l'Approche Communicative que dans ceux de l'Approche Actionnelle. Le texte littéraire pourrait être utilisé davantage pour apprendre aux élèves à bien lire à haute voix. L'élève/lecteur peut se transformer en lecteur/acteur au sens propre et figuré lorsqu'il lit un texte littéraire et dans le cas des manuels présentés, une pièce de théâtre, ou un extrait de film. Au sens figuré, puisqu'il interagit avec le texte, il pénètre dans le texte, il élucide des caractères, des sentiments et des comportements, il les filtre dans son crible psychologique et sentimental, il change de personnalité, pour adopter ensuite des intonations et des gestes adéquats qui le transformeront en un Autre, en un Acteur au sens propre du terme. L'élève lit silencieusement, comprend, ressent, filtre, se transforme, lit à haute voix dans un objectif communicatif précis, dernière étape avant de jouer son rôle en public. Le texte littéraire et plus particulièrement les pièces de théâtre et les poèmes, dans l'Approche Actionnelle, sont orientés vers un apprentissage des stratégies de lecture adaptées à la lecture de ces textes. Au moyen de projets de mise en scène et de récitation théâtrale les auteurs incitent implicitement à une lecture expressive préalable qui tient compte d'une compétence phonologique requise. Toutes les activités qui encadrent ce type de textes ont pour objectif le développement de l'accentuation et de l'intonation expressive.

L'élève/lecteur ressent, interprète, lit, communique un sentiment, un comportement, une émotion, donne aux graphèmes du relief, les transforment en mélodie porteuse de sens.

Ce type de lecture dans laquelle l'élève s'investit corps et âme ne peut être, sûrement pas, qualifié de lecture mécanique. Meta Lah mentionne 
(2013 : 204) que la lecture à haute voix n'est pas communicative. Pour nous, la lecture à haute voix n'est pas communicative dans la mesure où elle n'est pas expressive. Ce qui importe c'est comment on communique et de quels outils on se sert pour le faire. Ce qui nous amène à réfléchir sur le rôle de la compétence phonologique et particulièrement de la composante prosodique dans l'action de lire, une composante qui devrait être davantage travaillée en classe de FLE.

En tant que formateurs de formateurs, nous savons que les enseignants ont le guide pédagogique comme outil de formation. Nous espérions donc pouvoir y trouver des activités et une formation à l'enseignement de la lecture. Toutefois, d'après notre enquête, nous n'avons pas trouvé de conseils précis et clairs qui guident les enseignants sur la manière d'enseigner ce type de lecture.

Enfin, dans les manuels relevant de l'AA, adressés à un niveau B1, public grands adolescents qui ont servi de base à notre recherche, nous espérions trouver plus fréquemment des activités de lecture à haute voix et des directives pour l'enseignement de cette compétence ce qui n'a pas été vérifié par notre enquête et qui justifie ainsi le fait que plus le niveau monte, moins les enseignants proposent des activités de lecture à haute voix.

Éventuellement, les auteurs des manuels se conforment, à tort à notre avis, aux prescriptions du CECRL considérant la lecture à haute voix comme une activité mécanique et la bannissent des objectifs d'enseignement et des activités de leurs manuels.

Nous clôturerons notre étude en allant un peu plus loin que Meta Lah (2013 : 204) quand elle dit que « la question qui se pose est comment introduire la lecture à haute voix en classe, comment corriger la prononciation et surtout comment faire de cette activité une activité utile ». Il serait souhaitable que, dans les manuels de FLE à venir, le développement de la compétence lectorale devienne un objectif d'enseignement pour tous les niveaux, que la lecture à haute voix soit introduite dans les classes de langue aussi bien pour le développement de la production orale en général que pour celui de la compétence phonologique en particulier et qu'il y ait plus de conseils quant à la façon de l'enseigner dans les guides pédagogiques. Lire à haute voix, comme nous l'avons vu plus haut, peut être une activité motivante par son aspect théâtral et donc utile pour l'apprentissage d'une langue étrangère et de ses composantes langagières et communicatives. Elle est également utile puisque grâce à elle l'enseignant peut faire développer et corriger la prononciation de ses élèves au moyen d'activités diverses et variées portant sur les faits segmentaux et surtout suprasegmentaux. L'activité de lecture à haute voix devient ainsi entre les mains de l'enseignant en même temps moyen et objectif d'enseignement. On fait lire pour enseigner à bien prononcer et on fait bien prononcer pour enseigner à bien lire. 


\section{Bibliographie}

BEAUME 1987 : BEAUME, Edmond. « La lecture à haute voix ». Les actes de lecture $\mathrm{n}^{\circ} 18$ (1987).

CUQ, GRUCA 2005 : CUQ, Jean-Pierre et Isabelle GRUCA. Cours de didactique du français langue étrangère et seconde. Grenoble : PUG, 2005.

GIRARDET, CRIDLIG 1996 : GIRARDET, Jacky et Jean-Marie CRIDLIG. Panorama 2. Paris : CLE International, 1996.

GIRARDET, PÉCHEUR et al. 2004 : GIRARDET Jacky et Jacques PÉCHEUR, Colette GIBBE, Marie-Louise PARIZET. Tendances B1 : Méthode de français. Paris : CLE International, 2016.

GIRARDET, PÉCHEUR 2013 : GIRARDET, Jacky et Jacques PÉCHEUR. Écho B1.2 : Méthode de français. Paris : CLE International, 2013.

GIRARDET, PÉCHEUR 2014 : GIRARDET, Jacky et Jacques PÉCHEUR. Écho B1.1 : Méthode de français. Paris : CLE International, 2014.

GOIGOUX 2004 : GOIGOUX, Roland. " Méthodes et pratiques d'enseignement de la lecture ». L'apprentissage de la lecture $\mathrm{n}^{\circ} 1$ (2004) : 37-56. <http://www.revuedeshep.ch/site-fpeq-n/Site_FPEQ/1_ files/2005-1-Goigoux.pdf $>$ [consulté le 19/9/2019].

HEU, ABOU-SAMRA et al. 2015 : HEU, Elodie et Myriam ABOU-SAMRA, Marion PERRARD, Céline PINSON. LE NOUVEL édito B1 : Méthode de français. Paris : Didier, 2015.

LAH 2013 : LAH, Meta. « La lecture à haute voix en classe de langue étrangère - une activité à réhabiliter ? ». Linguistica, vol. 53, $\mathrm{n}^{\circ} 1$ (2013) : 199-207.

MORAZ, PRIKHODKINE 2011 : MORAZ, Myriam et Alexei PRIKHODKINE. " Phonétique et approche actionnelle : une mise en pratique ». A contrario no 15 (2011) : 99-116. <https://www.cairn.info/ revue-a-contrario-2011-1-page-99.htm.>

REYZABAL 1994 : REYZABAL, Maria-Victoria. « La lecture à voix haute. Technique pour la compréhension active des textes ». Revue internationale d'éducation de Sèvres no 2 (1994) : 83-86.

RIQUOIS 2010 : RIQUOIS, Estelle. « Exploitation pédagogique du texte littéraire et lecture littéraire en FLE : un équilibre fragile ». $11^{\text {es }}$ Rencontres des chercheurs en didactique des littératures, Genève (2010) : 247-251. $<$ https://www.unige.ch/litteratures2010/contributions_files/Riquois\%20 2010.pdf. $>$

RIQUOIS 2019 : RIQUOIS, Estelle. Lire et comprendre en français langue étrangère. Paris : Hachette Livre, 2019. 
Мариа (Маро) Ж. Патели

\section{ОД КОМУНИКАТИВНОГ ДО АКЦИОНОГ ПРИСТУПА: ВЕШТИНА ЧИТАҢА НАГЛАС У УЏБЕНИЦИМА}

У оквиру овог чланка настојали смо да испитамо постојање активности за развој вештине читања наглас у уџбеницима заснованим на комуникативном и акционом приступу. Истраживали смо да ли је и на који начин компетенција читања присутна у датим уџбеницима, да ли обухвата активност читања наглас, као и то које су врсте активности понуђене у педагошким приручницима и какви се методолошки савети у њима дају ради укључивања читања наглас. Резултати до којих смо дошли показали су да је овакав тип читања веома ретко експлицитно представљен у уџбеницима поменутих приступа и подразумева искључиво коришћење одломака књижевних дела.

Кључне речи: експресивно читање, комуникативни приступ, акциони приступ, уџбеници, актуелно стање, фонолошка вештина, супрасегментални елементи 